\title{
Influência do Zumbido no Índice Percentual de Reconhecimento de Fala em Pacientes Normo-ouvintes
}

\section{Influence of Tinnitus Percentage Index of Speech Recognition in Patients with Normal Hearing}

\author{
Daila Urnau*, Paula Andreta Barros da Silva*, Lílian Seligman**.
}

\author{
* Pós-graduanda em Distúrbios da Comunicação Humana da Universidade Federal de Santa Maria (UFSM), Santa Maria / RS, Brasil. Fonoaudióloga. \\ ** Doutora Professora Adjunta do Curso de Fonoaudiologia da Universidade Federal de Santa Maria (UFSM), Santa Maria / RS, Brasil. \\ Instituição: Universidade Federal de Santa Maria / RS. \\ Santa Maria / RS - Brasil. \\ Endereço para correspondência: Daila Urnau - Rua Vale Machado, 1605- Apto. 405 - Centro - Santa Maria / RS - Brasil - CEP: $97010-530$ - Telefone: (+55 55) \\ 3028-4070 / (+55 54) 9135-5594 - E-mail: daila_urnau@yahoo.com.br \\ Artigo recebido em 2 de Agosto de 2010. Artigo aprovado em $1^{\circ}$ de Setembro de 2010.
}

\section{RESUMO}

Introdução:

Objetivo:

Método:

Resultados:

Conclusão:

Palavras-chave:

$\overline{\text { SUMMARY }}$

Introduction:

Objective:

Methods:

Results:

Conclusion:

Keywords:
A compreensão da fala é um dos aspectos mensuráveis mais importantes da função auditiva humana. O zumbido prejudica a qualidade de vida, prejudicando a comunicação.

Investigar possíveis alterações no Índice Percentual de Reconhecimento de Fala (IPRF) em indivíduos portadores de zumbido que apresentem audição normal e analisar a relação entre zumbido, gênero e idade.

Estudo retrospectivo, através da análise de prontuários de 82 indivíduos de ambos os gêneros, com idade entre 21 a 70 anos, totalizando 128 orelhas com audição normal. As orelhas foram analisadas separadamente, e divididas em grupo controle, sem queixas de zumbido, e grupo estudo, com queixas de zumbido. Foram analisadas as variáveis gênero e faixa etária dos grupos e verificada a influência do zumbido no IPRF. Considerou-se normal, o percentual de 100\% de acertos, e alterado, o valor entre 88 a 96\%. Foram adotados estes critérios, já que o percentual inferior a 88\% de acertos é encontrado em indivíduos com perdas auditivas neurossensoriais.

Não se observou diferença estatisticamente significante entre as variáveis faixa etária e zumbido, IPRF e zumbido, somente entre gênero e zumbido. Encontrou-se prevalência de zumbido no gênero feminino (56\%), maior ocorrência de zumbido na faixa etária de 31 a 40 anos (41,67\%) e menor ocorrência entre 41 a 50 anos (18,75\%) e quanto ao IPRF, houve maior percentual de alterado em indivíduos portadores de zumbido $(61,11 \%)$.

O zumbido não interfere no IPRF e não há relação entre zumbido e idade, somente entre zumbido e gênero.

audição, zumbido, inteligibilidade da fala, audiometria da fala, grupos etários, distribuição por sexo.

The understanding of speech is one of the most important measurable aspects of human auditory function. Tinnitus affects the quality of life, impairing communication.

To investigate possible changes in the Percentage Index of Speech Recognition (SDT) in individuals with tinnitus have normal hearing and examining the relationship between tinnitus, gender and age. A retrospective study by analyzing the records of 82 individuals of both genders, aged 21-70 years, totaling 128 ears with normal hearing. The ears were analyzed separately, and divided into control group, no complaints of tinnitus and group study, with complaints of tinnitus. The variables gender and age groups and examined the influence of tinnitus in the SDT. It was considered normal, the percentage of $100 \%$ correct and changed, and the value between $88-96 \%$. These criteria were adopted, since the percentage below $88 \%$ correct is found in individuals with sensorineural hearing loss.

There was no statistically significant difference between the variables age and tinnitus, and tinnitus SDT, only gender and tinnitus. The prevalence of tinnitus in females (56\%), higher incidence of tinnitus in the age group 31-40 years (41.67\%) and fewer from 41 to 50 years (18.75\%) and on the SDT there was a greater percentage change in individuals with tinnitus (61.11\%).

The buzz does not interfere with SDT and there is no relationship between tinnitus and age, only between tinnitus and gender.

hearing, tinnitus, speech intelligibility, speech audiometry, age groups, sex distribution. 


\section{INTRODUÇÃO}

Existe uma forte correlação entre zumbido e perda auditiva (1), sendo que o zumbido é encontrado em 65\% das perdas auditivas neurossensoriais, $5 \%$ das perdas mistas e $4 \%$ das perdas condutivas, porém há dados que mostram a presença de zumbido também em indivíduos com audição normal (2). No último caso, a presença isolada do zumbido pode ser o primeiro sintoma de alguma patologia que somente será diagnosticada depois do aparecimento da perda auditiva.

O zumbido não é caracterizado como uma doença, e sim como um sintoma, no entanto, afeta milhares de pessoas no mundo inteiro (3).

A compreensão da fala é um dos aspectos mensuráveis mais importantes da função auditiva humana (4). O zumbido prejudica a qualidade de vida, causando prejuízo na comunicação (5).

Para um diagnóstico audiológico completo, é imprescindível a realização das medidas de reconhecimento de fala (4). O Índice Percentual de Reconhecimento de Fala (IPRF) tem por objetivo medir a inteligibilidade da fala, sendo realizado em uma intensidade fixa e confortável ao paciente, o qual deve repetir o maior número de palavras monossilábicas corretamente $(6,7)$. Pela análise do IPRF, pode-se observar o grau de dificuldade para a compreensão da fala. É considerado resultado dentro do esperado, quando o percentual for de 88 a 100\% de acertos, sendo a intensidade apresentada a $40 \mathrm{~dB}$ acima da média tritonal (500, 1000 e $2000 \mathrm{~Hz})(6)$.

A presença do zumbido e o baixo IPRF podem gerar alterações significativas na comunicação do indivíduo e consequentemente na qualidade de vida. O objetivo desse estudo é investigar possíveis alterações no IPRF em indivíduos portadores de zumbido que apresentem audição normal e analisar a relação entre zumbido, gênero e idade.

\section{MÉTODO}

Foi realizada uma pesquisa de natureza exploratória, com abordagem do problema de forma quantitativa, abrangendo atividades bibliográficas e análise de 480 avaliações audiológicas do banco de dados do Ambulatório de Audiologia do Hospital Universitário de Santa Maria (HUSM) atendidos nos anos de 2005, 2006, 2007 e de janeiro a agosto de 2008.

Os critérios de inclusão para esta pesquisa foram: apresentar avaliação audiológica normal, com presença ou ausência de queixas de zumbido referidas na anamnese audiológica e integridade do sistema tímpano-ossicular verificada através da imitanciometria (timpanograma de curva tipo A e reflexos acústicos presentes). Foi considerada avaliação audiológica normal as que apresentaram limiares auditivos iguais ou inferiores a $25 \mathrm{~dB}$ nas frequências de 250 a $8000 \mathrm{~Hz}$ (8).

A avaliação audiológica foi constituída por:

- Audiometria tonal limiar (ATL);

- Logoaudiometria (Limiar de Recepção de Fala - LRF e Índice Percentual de Reconhecimento de Fala - IPRF);

- Imitanciometria (Timpanometria e Reflexos Acústicos).

O Índice Percentual de Reconhecimento de Fala (IPRF) é a medida da inteligibilidade da fala em uma intensidade fixa na qual o indivíduo consegue repetir corretamente o maior número de palavras. Utiliza-se uma lista de 50 palavras monossílabicas, sendo apresentadas 25 palavras em cada orelha, com intensidade de $40 \mathrm{dBNS}$. Espera-se que indivíduos com audição normal apresentem entre 92 a 100\%, ou seja, o individuo poderá apresentar até dois erros (7).

Das 480 avaliações audiológicas analisadas, somente 82 estavam de acordo com os critérios de inclusão. Essas avaliações foram divididas em: Grupo Controle (GC), composto por indivíduos sem queixa de zumbido e Grupo Estudo (GE), composto por indivíduos com queixa de zumbido, porém, nesse estudo, foram analisadas as orelhas separadamente dentro dos critérios supracitados. Os dois grupos foram compostos por indivíduos de ambos os sexos.

A idade da amostra variou entre 21 a 70 anos de idade. Os pacientes foram classificados em cinco faixas etárias:

- Faixa 1: entre 21 a 30 anos;

- Faixa 2: entre 31 a 40 anos;

- Faixa 3: entre 41 a 50 anos;

- Faixa 4: entre 51 a 60 anos;

- Faixa 5: entre 61 a 70 anos.

Foram analisadas estatisticamente as variáveis gênero e zumbido, faixa etária e zumbido, assim como a influência do zumbido no IPRF do Grupo Estudo.

Para análise estatística, esse estudo classificou os resultados do IPRF como, IPRF sem influência, quando o percentual obtido foi de 100\% de acertos e como IPRF com influência, quando o valor encontrado foi 92 e 96\%. Foram adotados estes critérios, já que o estudo foi composto apenas de indivíduos normo-ouvintes, ou seja, que apresentaram IPRF acima de 88\%. O percentual inferior a 88\% 
de acertos é encontrado em indivíduos com perdas auditivas neurossensoriais (7).

Os grupos foram analisados estatisticamente através dos testes não-paramétricos qui-quadrado e teste de diferença entre duas proporções, sendo o valor de significância de 0,05 (5\%), o que é estatisticamente significante quando o valor de significância calculada $(p)$ for menor do que o nível de significância adotado.

O presente estudo está vinculado ao Projeto "Pesquisa e base de dados em saúde auditiva", estando devidamente registrado no Comitê de Ética em Pesquisa, sob o número 0138.0.243.246-06.

\section{RESULTADOS}

Pelos critérios de inclusão, foram selecionados 82 indivíduos, totalizando 128 orelhas com audição normal. Para este estudo foram analisadas as orelhas separadamente, dentro dos critérios de inclusão, pois cada orelha apresenta comportamento estatisticamente independente.

O grupo controle (GC) foi composto por 56 orelhas, de pacientes com idade entre 22 e 66 anos, 42 do sexo feminino e 14 do sexo masculino.

O grupo estudo (GE) foi composto de 72 orelhas, de pacientes com idade de 21 a 70 anos, 40 orelhas do sexo feminino e 32 do sexo masculino.

Dos 480 prontuários, 17,08\% apresentaram audição normal e 7,5\% apresentaram queixas de zumbido, sendo ambos analisados neste estudo.

Houve prevalência do gênero feminino em ambos os grupos, com percentual de $56 \%$ no grupo estudo e $75 \%$ no grupo controle (Gráfico 1). Com relação ao gênero e a presença ou ausência de zumbido, foi encontrada diferença significativa, através do teste qui-quadrado $(p=0,023)$. Esta diferença encontrada deve-se ao elevado número de indivíduos do sexo feminino no grupo controle.

Foi utilizado o teste qui-quadrado para comparar a variável gênero com IPRF sem influência e IPRF com influência, porém não houve diferença significante $(p=0,787)$, no entanto, nota-se que houve maior número de IPRF com influência no gênero feminino (Tabela 1 ).

Entre os indivíduos do GE (com queixas de zumbido), observou-se que a faixa etária de 31 a 40 anos (faixa 2) apresentou maior ocorrência de zumbido (41,67\%) (Gráfico 2), porém, a média de idade dos indivíduos com IPRF sem
Tabela I. Gênero XIPRF.

\begin{tabular}{lccc}
\hline Gênero & $\begin{array}{c}\text { IPRF sem } \\
\text { influência }\end{array}$ & $\begin{array}{c}\text { IPRF com } \\
\text { influência }\end{array}$ & p-valor \\
\hline Feminino & 15 & 25 & 0,787 \\
Masculino & 13 & 19 & \\
\hline
\end{tabular}

*Teste estatistico do qui-quadrado

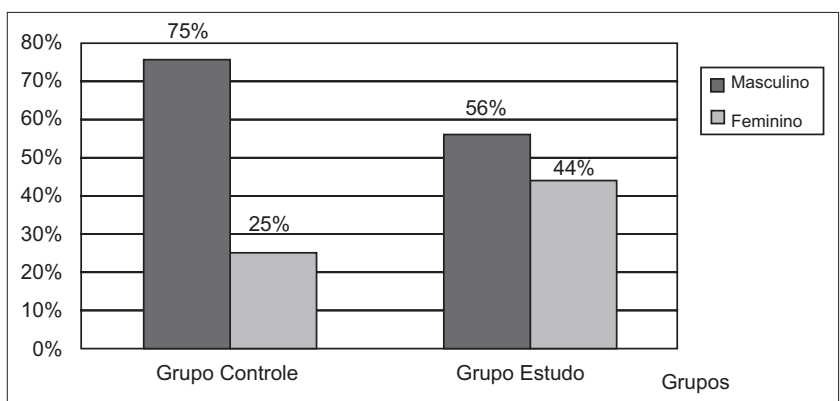

Gráfico 1. Indivíduos distribuídos quanto à variável gênero.

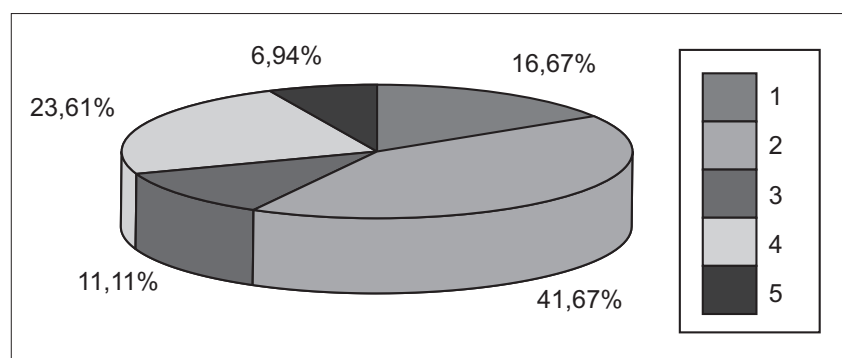

Gráfico 2. Amostra de indivíduos com zumbido distribuídos quanto à variável faixa etária.

influência (41,43 anos) e IPRF com influência (41,57 anos) encontra-se na faixa etária de 41 a 50 anos (faixa 3).

Comparando as faixas etárias da amostra, observouse que a faixa etária que menos apresentou queixa de zumbido foi a de 41 a 50 anos (faixa 3), com 18,75\%. A que apresentou mais queixas de zumbido, foi a faixa 2 (de 31 a 40 anos), com 23,44\%, concordando com o gráfico anterior (Gráfico 2). A faixa etária 5 (de 61 a 70 anos) foi a que apresentou menor número de orelhas em ambos os grupos (Gráfico 3).

Analisando as variáveis zumbido e IPRF de ambos os grupos, não se observou diferença estatisticamente significante, quando aplicado o teste do qui-quadrado $(p=0,20)$. Porém, verificou-se que há maior percentual de IPRF com influência em pacientes portadores de zumbido (61,11\%) em relação a não-portadores (50\%). Comparando ainda, os IPRF com influência de ambos os grupos, através do teste para diferença entre duas proporções $(p=0,21)$ não se observou diferença estatisticamente significante (Gráfico 4). 


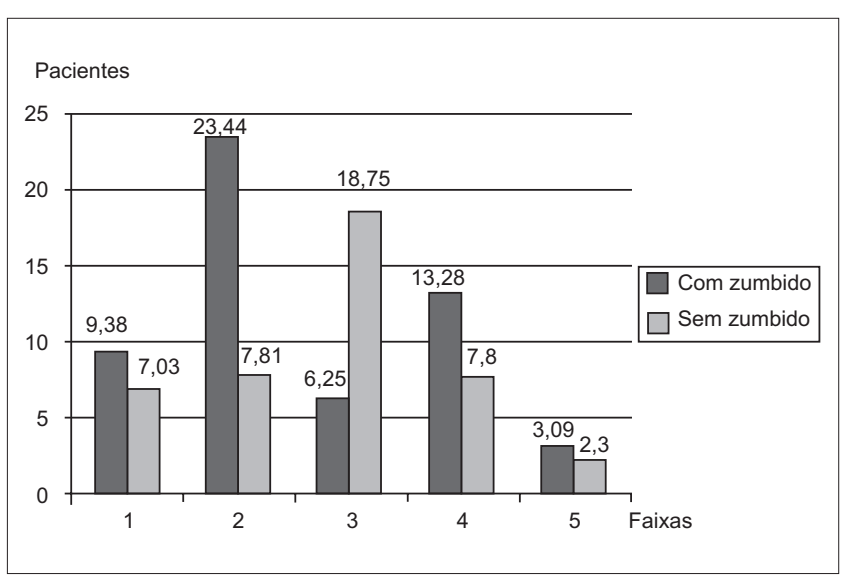

Gráfico 3. Faixa etária x Número de pacientes.

\section{DISCUSSÃO}

De acordo com o National Institute of Health, o zumbido é um sintoma muito frequente, afetando cerca de 15\% dos americanos (9). Um estudo (10) a respeito da prevalência do zumbido em uma amostra aleatória de uma capital brasileira, concluiu que $25 \%$ dos pesquisados apresentaram essa queixa, independente da configuração audiométrica apresentada. Porém, neste estudo, com apenas indivíduos com audição normal, 7,5\% do total de indivíduos atendidos no período estudado, apresentaram queixas de zumbido. Este achado concorda com a literatura, que refere $(1,11)$ percentual muito próximo a este $(7,4 \%$ e 8 a $10 \%)$.

O gênero feminino prevaleceu nos dois grupos (Gráfico 1), sendo encontrada diferença estatisticamente significante entre os grupos. Estes achados corroboram com outro estudo (1) semelhante, no qual a proporção de mulheres também foi maior em ambos os grupos, (67,3\% no grupo de estudo e 55,6\% no grupo controle), porém não houve diferença significante entre os grupos.

O estudo abrangeu uma extensa faixa etária ( 21 a 66 anos). Para alguns autores (12) o zumbido pode acometer todas as faixas etárias. A faixa etária que apresentou maior número de indivíduos portadores de zumbido foi a de 31 a 40 anos (41,67\%). Resultado semelhante a esse foi encontrado na literatura (13), a qual refere maior número de indivíduos com idade abaixo de 40 anos (46,6\%).

Algumas pesquisas $(1,14)$ afirmam que o zumbido aparece após os 40 anos e outro estudo referiu média de idade do grupo com queixa de zumbido de 45,62 anos (14). Contrariando esses estudos, verificou-se que indivíduos entre 41 a 50 anos (faixa 3) apresentaram menos queixas de zumbido.

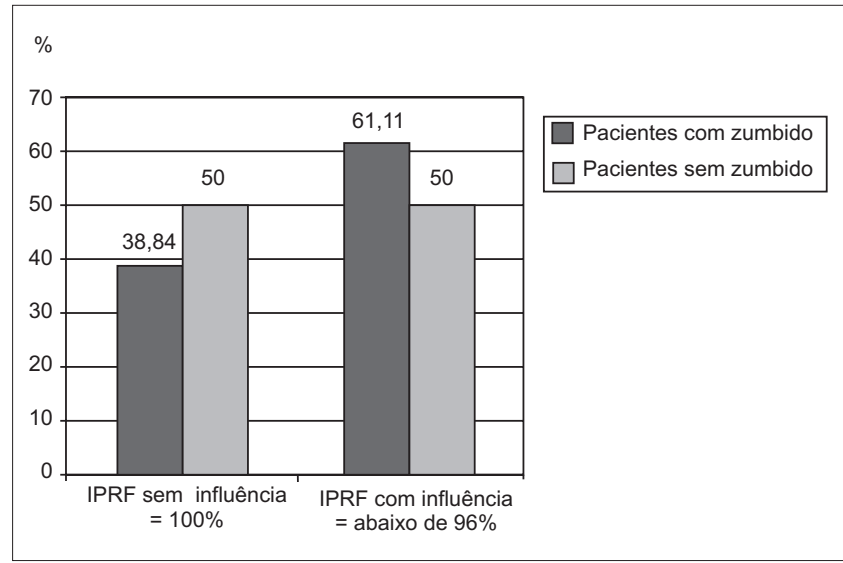

Gráfico 4. Percentual de pacientes x IPRF.

Com relação à idade, encontraram-se poucos indivíduos na faixa de 61 a 70 anos em ambos os grupos. Acredita-se que este número reduzido de indivíduos devese à alta incidência de perda auditiva nesta faixa etária, a qual não se inclui nos objetivos desse estudo. Há predomínio de perda auditiva em pacientes idosos, geralmente com média de idade igual a 65 anos (2). A literatura (15) ainda aponta o aumento do zumbido com o aumento da idade.

Cerca de 95\% da população normal já sentiu zumbido, mas ele é considerado importante quando se torna contínuo e incomodativo (16). Os casos de zumbido em indivíduos com audição normal constituem uma amostra muito importante, pois suas características podem ser atribuídas exclusivamente ao zumbido, e não à perda auditiva (17). Nesses casos, o impacto é maior, causando ansiedade, irritação, tensão, insônia e, algumas vezes, até depressão $(18,19)$.

O paciente portador de zumbido deve sempre ser submetido à avaliação médica para diagnóstico e tratamento de possível patologia de base (20). Acredita-se que nos indivíduos acometidos, ocorra uma perda da modulação das células ciliadas externas com as células ciliadas internas normais, gerando uma atividade anormal das vias auditivas, interpretadas de forma errônea como um som. As células ciliadas externas mais susceptíveis à lesão são aquelas localizadas na região mais basal da cóclea. Outra hipótese para a ocorrência de zumbido em indivíduos sem perda auditiva, poderia ser explicada pelo dano difuso de até 30\% das células ciliadas externas em toda a espiral da cóclea, sem haver o comprometimento do limiar auditivo (3).

Em vista disso e considerando que, o zumbido é na maioria dos casos, um problema debilitante (21) podendo levar até o portador à tentativa de suicídio (22), ressalta-se a importância da investigação sobre o zumbido. 
Sua causa ainda é indefinida, porém existem inúmeras hipóteses. Pode ser um sintoma de algumas patologias, tais como: otológicas, metabólicas, psiquiátricas, odontológicas, traumáticas, cardiovasculares, neoplásicas, neurológicas e farmacológicas (9, 23,24).

Sabe-se que o zumbido é um transtorno que produz incômodo na vida social, no desempenho profissional e até mesmo na relação familiar (25). Na maioria das vezes, pode tornar-se intolerável, passando a interferir no sono, na concentração e no equilíbrio emocional (26).

Houve maior número de IPRF com influência no gênero feminino, provavelmente devido a prevalência desde gênero nos dois grupos estudados. A idade média dos indivíduos de IPRF com influência encontrou-se na faixa etária de 41 a 50 anos (faixa 3). Uma das possíveis explicações para esta piora no reconhecimento da fala, principalmente nessa faixa etária, seria o início do efeito do envelhecimento, além da influência do zumbido. Um estudo (27) sobre envelhecimento e reconhecimento de fala em indivíduos normo-ouvintes, pela análise dos limiares de sentenças no silêncio e no ruído, constatou que o reconhecimento de fala de indivíduos com mais de 50 anos foi significativamente inferior a de adultos jovens.

Não se encontraram estudos que relacionam zumbido e os testes de fala. No estudo realizado, não foi encontrada relação significativa entre a presença de zumbido e a diminuição do IPRF em pacientes com audição normal.

Há na literatura relatos (28) correlacionando zumbido e dificuldade de compreensão de fala em indivíduos com queixa de perda auditiva, sendo escassos os estudos em indivíduos normo-ouvintes.

\section{ConCluSÃO}

Concluiu-se que a presença de zumbido não interfere na inteligibilidade da fala, analisada através do IPRF, bem como não há relação entre zumbido e idade. Encontrou-se relação entre zumbido e o gênero feminino.

Devido aos resultados encontrados nessa pesquisa e da pouca literatura encontrada, sugerem-se mais estudos com amostras maiores sobre o presente assunto.

\section{REFERÊNCIAS BIBLIOGRAFICAS}

1. Sanchez TG, Medeiros IRT, Levy CPD, Ramalho JRO, Bento RF. Zumbido em pacientes com audição normal: caracterização clínica e repercussões. Rev Bras Otorrinolaringol. 2005, 71(4):427-31.

2. Moura LOS, Iorio MCM, Azevedo MF. A eficácia da adaptação da prótese auditiva na redução ou eliminação do zumbido. Rev Bras Otorrinolaringol. 2004, 70(5):624-631.

3. Figuerêdo RBS, Corona AP. Influência do zumbido nos limiares de altas freqüências. Rev Soc Bras Fonoaudiol. 2008, 12(1):29-33.

4. Caporali, SA, Silva JA. Reconhecimento de fala no ruído em jovens e idosos com perda auditiva. Rev Bras Otorrinolaringol. 2004, 70(4):525-32.

5. Silva RCF, Bandini HHM, Soares IM. Aparelho de amplificação sonora individual: melhora a sensação de zumbido? Rev CEFAC. 2007, 9(2):263-68.

6. Gómez MVSG, Pedalini MEB. Testes Audiológicos para a Identificação de Alterações Cocleares e Retrococleares. Em: Lopes Filho O. Tratado de Fonoaudiologia. São Paulo: Roca; 1997. p.127-47.

7. Russo I, Lopes LQ, Brunetto-Borgianni LM, Brasil LA. Logoaudiometria. In: Momensohn-Santos TM, Russo ICP. Prática da Audiologia Clínica. São Paulo:Cortez; 2007. p.13554.

8. Davis H, Silverman RS. Hearing and deafness. 3rd ed. New York: Holt, Rinehart \& Winston; 1970.

9. Sanchez TG, Ferrari GMS. O que é zumbido? Em: Samelli AG. Zumbido: avaliação, diagnóstico e reabilitação: abordagens atuais. São Paulo: Lovise; 2004. p.17-22.

10. Gomes SJV, Barboza RM, Santos TMM. A incidência de zumbido numa amostra aleatória na cidade de Salvador. Rev CEFAC. 2004, 6(1):89-93.

11. Barnea G, Attias J, Gold S, Shahar A. Tinnitus with normal hearing sensitivity: extended high-frequency audiometry and auditory nerve brain-stem-evoked responses. Audiology. 1990, 29:36-45.

12. Józefowicz-korczynska M, Pajor A. Evaluation of oculomotor tests in patients with tinnitus. Int Tinnitus J. 2002, 8(2):100-3.

13. Almeida LD, Mitre EI, Lemos L, Simões ECC. Vestibulometria em Indivíduos com Zumbido e Exames Audiológicos Normais. Rev CEFAC. 2005, 7(3):382-87.

14. Schneider D, Schneider L, Shulman A, Claussen CF, Just E, Koltchev C, et al. Gingko biloba (Rökan) therapy in tinnitus 
patients and measurable interactions between tinnitus and vestibular disturbances. Int Tinnitus J. 2000, 6(1):56-62.

15. Baguley DM. Mechanisms of tinnitus. Br Med Bull. 2002, 63:195-202.

16. Branco F. Tratamento fonoaudiológico do zumbido. Em: I Simpósio de atualização em audiologia da UFMG. Universidade Federal de Minas gerais. Minas Gerais: Belo Horizonte, 2003.

17. Sanchez TG, Medeiros IRT, Levy CPD, Ramalho JRO, Bento RF. Zumbido em pacientes com audiometria normal: caracterização clínica e repercussões. Rev Bras Otorrinolaringol. 2005, 71(4):427-31.

18. Mueller HG, Hall JW. Audiologist desk reference. Em: Audiologic management, rehabilitation, and terminology. San Diego:Singular, 1998. p.611-40.

19. Fukuda Y. Zumbido: diagnóstico e tratamento. Rev Bras Otorrinolaringol. 1997, 4(2):39-43.

20. Mor R, Azevedo MF. Emissões otoacústicas e sistema olivococlear medial: pacientes com zumbido sem perda auditiva. Pró-fono. 2005, 17(3):283-92.

21. Jastreboff P, Hazell JWP. A neurophysiological approach to tinnitus: clinical implications. Brit Journ of Audiol. 1993, 27:7-17.
22. Fukuda Y.Zumbido Neurossensorial. Rev Neurociências. 2000, 8(1):6-10.

23. Seidman MD, Jacobson GP. Update on tinnitus. Otoryngol Clin North Am. 1996, 29(3):455-65.

24. Sanchez TG, Bento RF, Miniti A, Câmara J. Zumbido: características e epidemiologia. Experiência do Hospital das Clínicas da Faculdade de Medicina da Universidade de São Paulo. Rev Bras Otorrinolaringol. 1997, 63(3):229-35.

25. Caovila HH, Silva MLG, Munhoz MSL, Ganança MM. Entendendo as tonturas: o que você precisa saber sobre os distúrbios do labirinto. São Paulo: Atheneu; 1999.

26. Sanchez TG. Zumbido: Análise crítica de uma experiência de pesquisa. São Paulo; 2003. (Tese de Livre-Docência, Faculdade de Medicina da Universidade de São Paulo).

27. Soncini F, Costa MJ, Oliveira TMT. Influência do processo de envelhecimento no reconhecimento da fala em indivíduos normo-ouvintes. Pró-fono. 2003, 15(3):287-296.

28. Moreira RR, Ferreira Junior M. Testes de fala: aplicação em portadores de perda auditiva induzida por ruído. Prófono. 2004, 16(3):293-300. 